\title{
Multispectral mid-infrared imaging using frequency upconversion
}

Sanders, Nicolai Højer; Dam, Jeppe Seidelin; Jensen, Ole Bjarlin; Tidemand-Lichtenberg, Peter; Pedersen, Christian

Published in:

Proceedings of SPIE - The International Society for Optical Engineering

Link to article, DOI:

$10.1117 / 12.2003947$

Publication date:

2013

Document Version

Publisher's PDF, also known as Version of record

Link back to DTU Orbit

Citation (APA):

Sanders, N. H., Dam, J. S., Jensen, O. B., Tidemand-Lichtenberg, P., \& Pedersen, C. (2013). Multispectral midinfrared imaging using frequency upconversion. Proceedings of SPIE - The International Society for Optical Engineering, 8604, [86040R]. https://doi.org/10.1117/12.2003947

\section{General rights}

Copyright and moral rights for the publications made accessible in the public portal are retained by the authors and/or other copyright owners and it is a condition of accessing publications that users recognise and abide by the legal requirements associated with these rights.

- Users may download and print one copy of any publication from the public portal for the purpose of private study or research.

- You may not further distribute the material or use it for any profit-making activity or commercial gain

- You may freely distribute the URL identifying the publication in the public portal 


\title{
Multispectral mid-infrared imaging using frequency upconversion
}

\author{
Nicolai Sanders, Jeppe Seidelin Dam*, Ole Bjarlin Jensen, Peter Tidemand-Lichtenberg, Christian \\ Pedersen
}

Technical University of Denmark, DTU Fotonik, Frederiksborgvej 399, 4000 Roskilde.

\begin{abstract}
It has recently been shown that it is possible to upconvert infrared images to the near infrared region with high quantum efficiency and low noise by three-wave mixing with a laser field [1]. If the mixing laser is single-frequency, the upconverted image is simply a band-pass filtered version of the infrared object field, with a bandwidth corresponding given by the acceptance parameter of the conversion process, and a center frequency given by the phase-match condition. Tuning of the phase-matched wavelengths has previously been demonstrated by changing the temperature [2] or angle [3] of the nonlinear material. Unfortunately, temperature tuning is slow, and angle tuning typically results in alignment issues. Here we present a novel approach where the wavelength of the mixing field is used as a tuning parameter, allowing for fast tuning and hence potentially fast image acquisition, paving the way for upconversion based real time multispectral imaging. In the present realization the upconversion module consists of an external cavity tapered diode laser in a Littrow configuration with a computer controlled feedback grating. The output from a tunable laser is used as seed for a fiber amplifier system, boosting the power to approx. $3 \mathrm{~W}$ over the tuning range from 1025 to $1085 \mathrm{~nm}$. Using a periodically poled lithium niobate crystal, the infrared wavelength that can be phase-matched is tunable over more than $200 \mathrm{~nm}$. Using a crystal with multiple poling periods allows for upconversion within the entire transparency range of the nonlinear material.
\end{abstract}

Keywords: Infrared imaging, nonlinear frequency conversion, diode lasers, upconversion

\section{INTRODUCTION}

\subsection{Infrared imaging}

Mid-infrared (Mid-IR) imaging and spectroscopy is interesting because many of the $\mathrm{C}-\mathrm{H}, \mathrm{C}-\mathrm{O}$ and $\mathrm{O}-\mathrm{H}$ bonds found in organic materials have absorption transitions in the 3 to $5 \mu \mathrm{m}$ range. This makes fast IR spectroscopy and imaging of weak signals useful for identification of various organic materials. By upconversion of the mid-IR light to near-infrared (NIR), we can use standard CCD's to detect it. This potentially improves the noise characteristics by several orders of magnitude over traditional IR detectors, and eliminates the need for cryogenic cooling.

The applications include early diagnosis of skin cancer, monitoring of food quality, detection of outgassing from explosives in roadside bombs, and other in situ IR imaging or spectroscopic applications.

${ }^{*}$ jdam@fotonik.dtu.dk

Nonlinear Frequency Generation and Conversion: Materials, Devices, and Applications XII edited by Konstantin L. Vodopyanov, Proc. of SPIE Vol. 8604, 86040R

(C) 2013 SPIE $\cdot$ CCC code: $0277-786 X / 13 / \$ 18 \cdot$ doi: $10.1117 / 12.2003947$

Proc. of SPIE Vol. 860486040 R-1 


\subsection{Prior work}

In previous publications we have described how mid-infrared light carrying image information can be frequency shifted to the NIR by mixing with a pump laser at $1064 \mathrm{~nm}$ [1]. The exact infrared wavelength that is upconverted depends on the angle through the crystal, which enables the setup to be used as an infrared spectrometer [4].

The upconverted wavelength in the center of the image can be changed by scanning of the crystal temperature, which enables the capture of multispectral images, containing a full spectrum for each position in the image. Unfortunately the speed of the temperature change is functionally limited by mechanical stresses between the crystal and its antireflection coating.

\subsection{This paper}

In the setup described in this paper, the phase-matched infrared wavelength is changed by scanning of the wavelength of the mixing laser, rather than the crystal temperature. This allows the upconverted wavelength to be tuned much faster, and over a wider range compared to temperature scanning, enabling fast imaging or spectroscopy over a wide range of mid-infrared wavelengths.

This paper covers some of the theory behind the frequency upconversion process. It describes the setup that was used to generate the tunable mixing light, used for frequency upconversion and capture of the images. Finally, we present some of the acquired images, showing emission lines of hot water vapor generated by the combustion in a candle, and a series of images showing the light emitted by a $700 \mathrm{~K}$ black body.

\section{SETUP DESCRIPTION}

The setup consists of a tunable pump laser, which is used for sum-frequency mixing with the mid-IR light in a periodically poled lithium niobate crystal (PPLN). The converted light is then captured by a Si-based CCD camera.

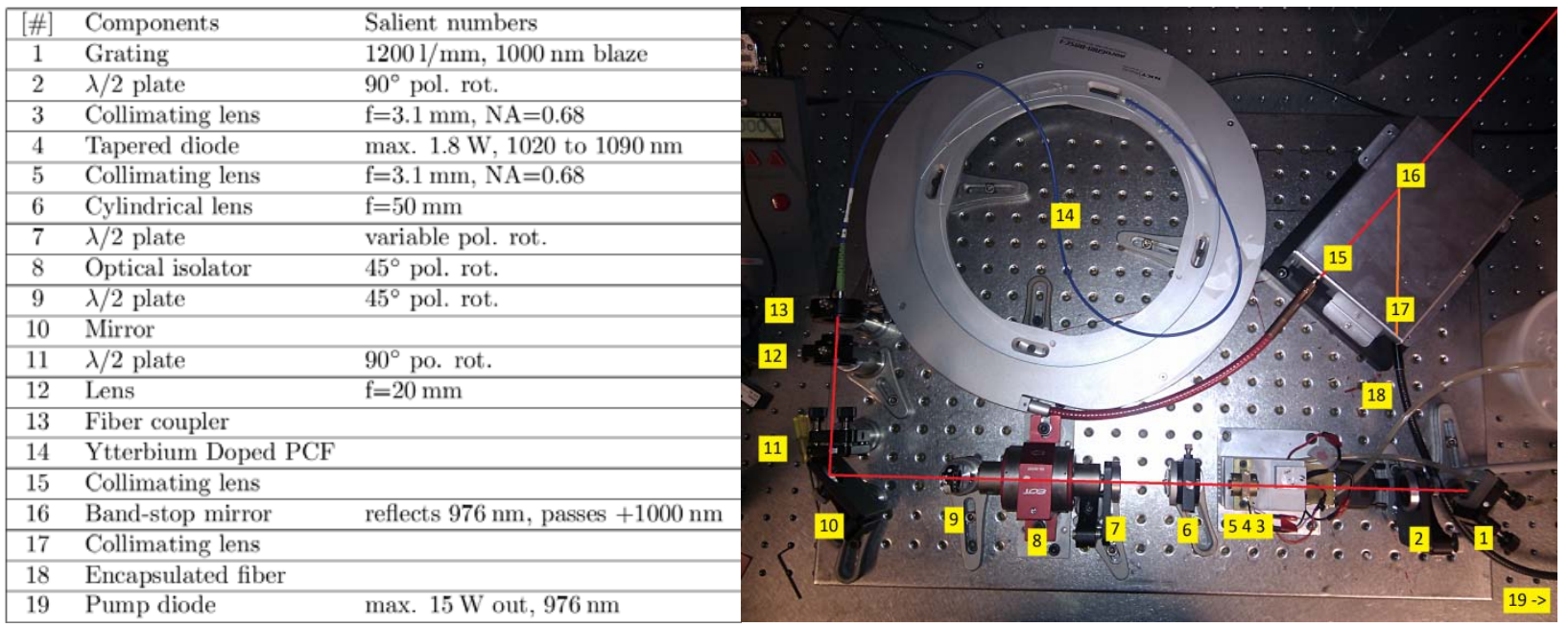

Figure 1. This is a picture of the pump laser, along with a numbered table of components. The power supplies and cooling pumps are not shown.

The pump laser consists of a tunable external cavity tapered diode laser (ECDL) with computer controlled grating feedback in the Littrow configuration. The tunable ECDL seeds an ytterbium doped photonic crystal fiber amplifier (DC200/40-PZ-Yb from NKT Photonics), which is pumped by a $12 \mathrm{~W}, 976 \mathrm{~nm}$ laser diode. The seed laser is tunable from 
$1020 \mathrm{~nm}$ to $1090 \mathrm{~nm}$, and generates between $400 \mathrm{~mW}$ and $1800 \mathrm{~mW}$ in within this tuning range. The output from the fiber amplifier is approximately $3 \mathrm{~W}$, linearly polarized, single-frequency, tunable from $1025 \mathrm{~nm}$ to $1085 \mathrm{~nm}$ oscillating in a perfect Gaussian mode.

The output from the fiber amplifier is single-passed through a periodically poled lithium niobate crystal; $40 \mathrm{~mm}$ long with AR coated end-facets and a poling period of $20.58 \mu \mathrm{m}$. The mid-IR light is filtered through a germanium window, to remove any visible light emitted by the object. Then the IR-signal is passed through a 45 degree mirror which is AR coated around $3 \mu \mathrm{m}$ and HR coated for 1020-1090 nm light. This mirror also directs the tunable mixing light into the crystal, see Fig. 2. The mid-IR light is converted to a wavelength around $800 \mathrm{~nm}$ in the PPLN crystal through sumfrequency mixing. After the nonlinear material the pump light is reflected by a second 45 degree mirror, HR coated at $1064 \mathrm{~nm}$ and AR coated around $800 \mathrm{~nm}$, allowing the upconverted light to pass. After this mirror three additional filters are used to remove ambient visible light and any remaining pump light; a long-pass filter with cutoff at $750 \mathrm{~nm}$, a shortpass filter at $850 \mathrm{~nm}$, and a short-pass filter at $1000 \mathrm{~nm}$. After these filters the upconverted light is imaged onto a CCD by a standard lens.

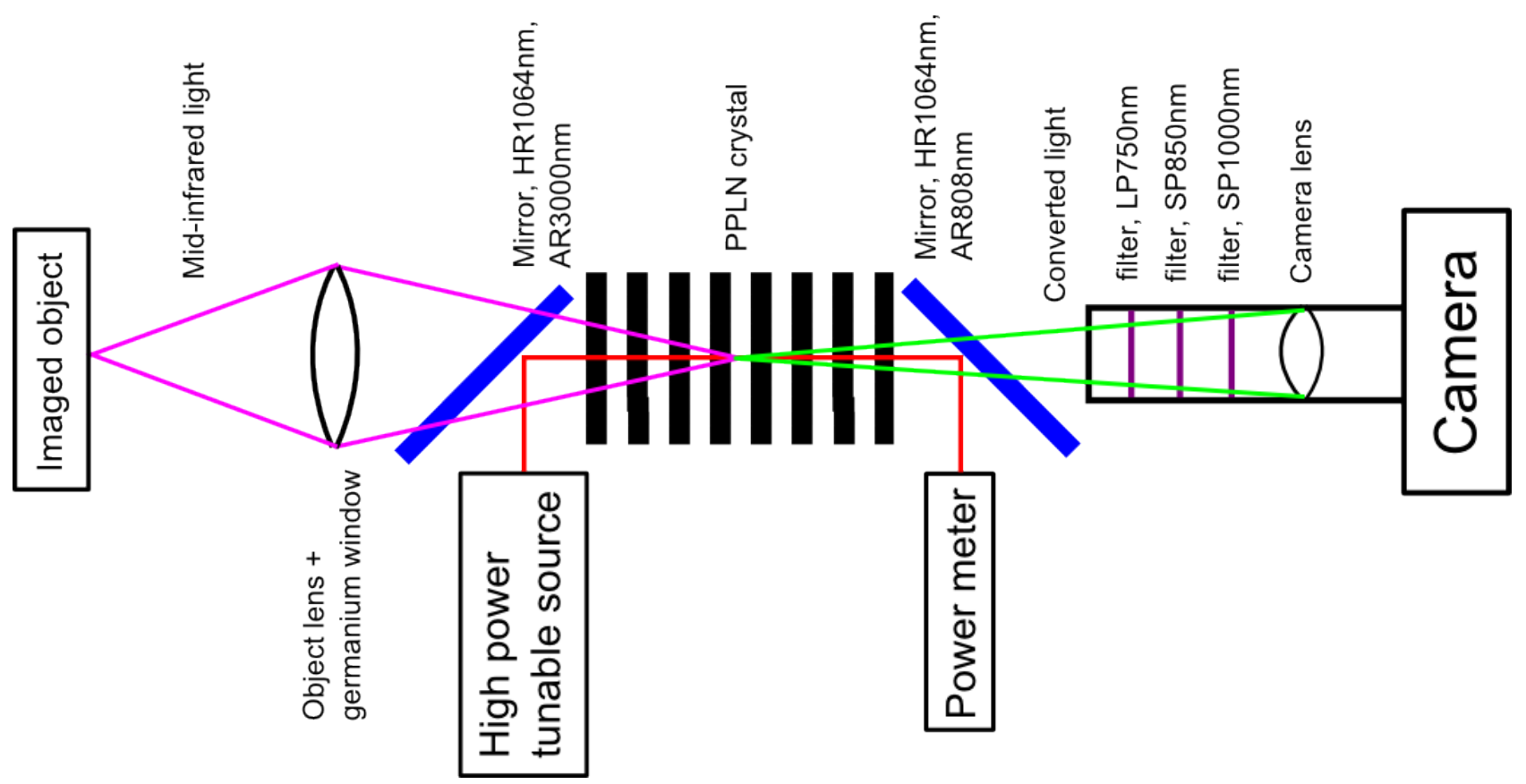

Figure 2. A diagram of the upconversion setup.

\section{THEORY}

\subsection{Phase-matching and conversion}

The phase-match condition arises because the light converted in different parts of the crystal must interfere constructively in order to make efficient conversion. This is equivalent to requiring that the photon momentum should be conserved during the conversion process. Also the photon energy is conserved during the conversion, i.e. the mid-IR photons is added the energy of one mixing photon. 
It is well-known that the phase matched wavelength depends on the poling period and pump wavelength for co-propagating beams. In image upconversion, however, it is important to consider non-collinear phase-matching between the mid-IR signal and the mixing field, as the upconversion process occurs in the Fourier plane, where the image information is coded in angles. A detailed analysis of the phase-matching properties of PPLN is beyond the scope of this paper, however it turns out that the phase-matched mid-IR wavelength, in addition to the angular dependence, can be scanned by tuning of the mixing laser wavelength, as shown in Fig. 3, as calculated using the Sellmeier equations in ref [5].

If images are acquired with mixing laser wavelengths spaced less than $0.3 \mathrm{~nm}$ from each other, it is possible to reconstruct the infrared spectrum for each point in the image, with equal representation of the acquired mid-IR spectrum. This is because the FWHM of the phase-matching is 0.3 to $0.6 \mathrm{~nm}$ for the mixing field, corresponding to a FWHM of 1.7 to $3.4 \mathrm{~nm}$ for the mid-IR wavelengths.

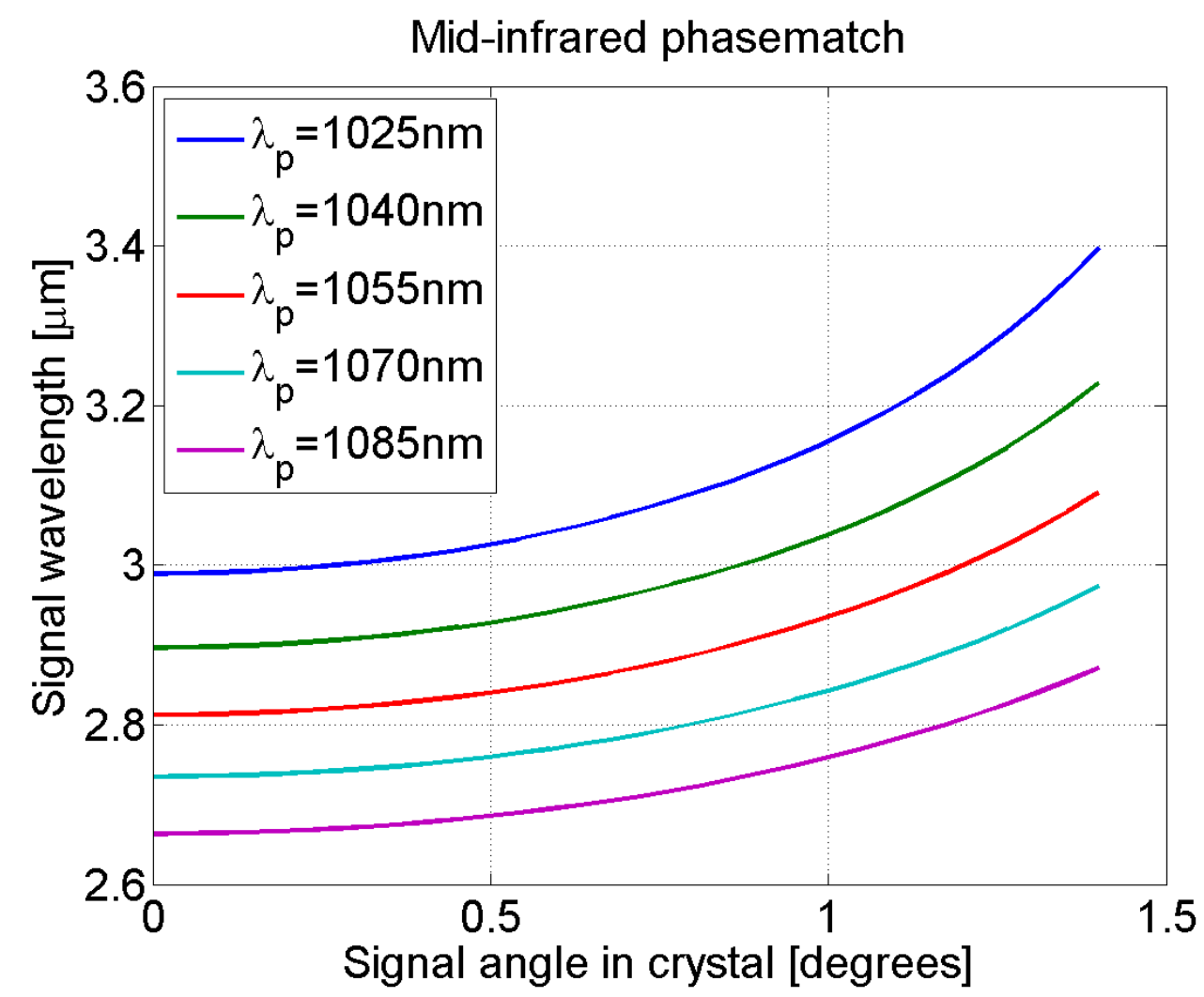

Figure 3. This picture shows the mid-IR wavelengths that are converted as function of angle through a PPLN crystal with a poling period of $20.58 \mu \mathrm{m}$ for five different pump wavelengths.

The quantum efficiency of the nonlinear upconversion in this setup is calculated to be approx. $2.5 \%$ using the theory derived in ref. [6]. 


\section{RESULTS}

In order to demonstrate the multispectral imaging capability of nonlinear image upconversion, series of pictures were obtained by tuning of the mixing laser. Figure 4 and 5 shows images of a candle and a soldering iron, respectively, for 6 equidistant pump wavelengths ranging from 1050.6 to $1053 \mathrm{~nm}$. One should focus on the changing position of the central emission line as the pump wavelength increases. The exposure times were $15 \mathrm{~s}$ for the images of the candle, to average out the flickering of the candle flame, and $10 \mathrm{~s}$ for the images of the soldering iron.

At the time of submission, our experimental data show the validity of the setup, and we expect to demonstrate some of the practical applications when a better mid-IR light source is available.

The first series shows the emission lines from hot water vapor produced when burning a candle. The images are not equally bright because the candle itself flickers during the $15 \mathrm{~s}$ exposure time. Nonetheless, it is easy to see that the emission lines forms expanding concentric circles as the mixing wavelength is increased.
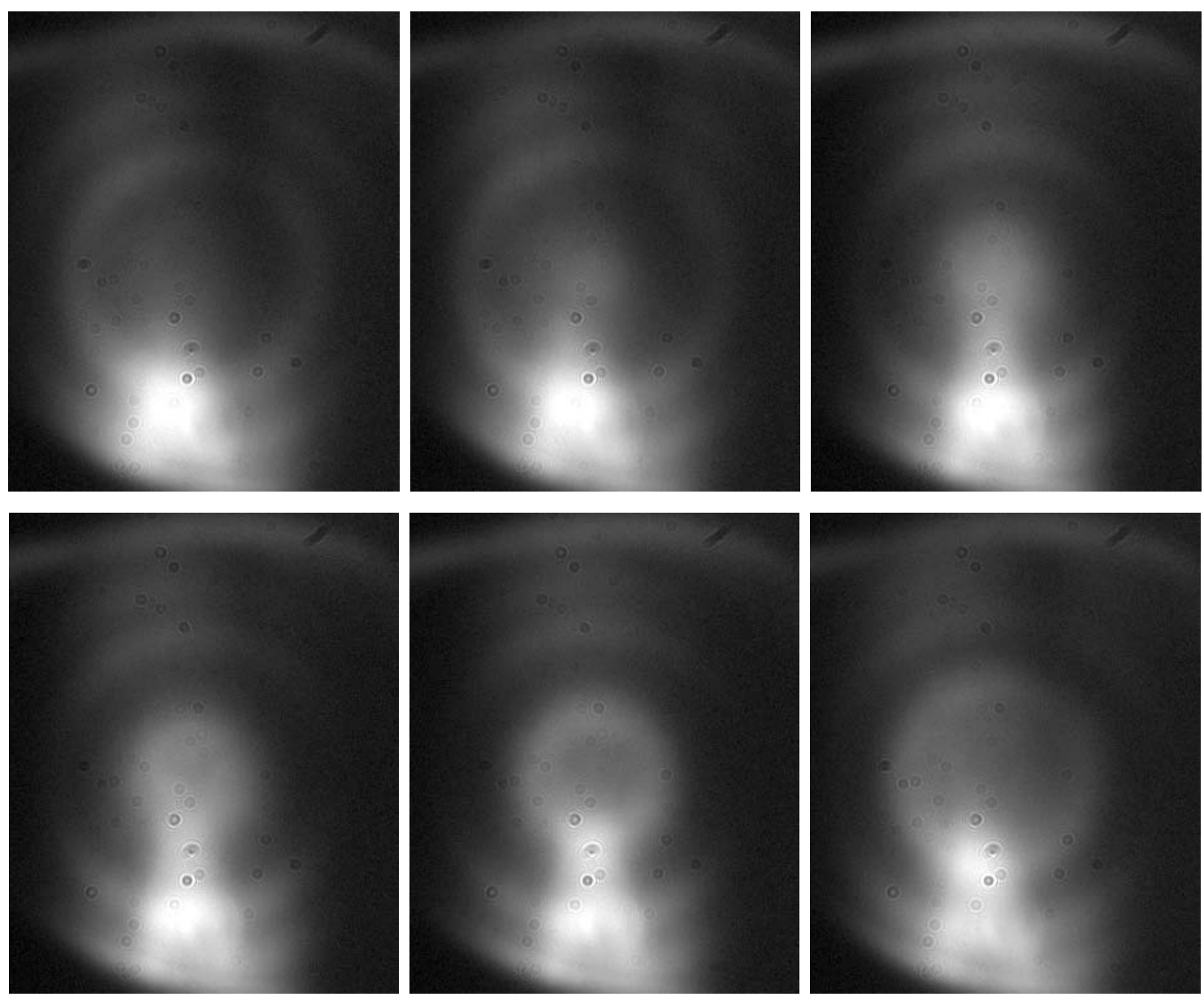

Figure 4. The concentric rings in these images are the emission lines from hot water vapor, taken with pump wavelengths from 1050.6 to $1053 \mathrm{~nm}$. These emission lines are positioned approx. $18 \mathrm{~nm}$ apart in the range from $2.8 \mu \mathrm{m}$ to $3 \mu \mathrm{m}$. They are converted according to their angle relative to the mixing laser, which is at the center of the rings. Tuning the mixing wavelength changes the position of the rings, which means that a full spectrum at each spatial position (pixel) can be extracted from a series of these images. 
The second series shows the emission from a $700 \mathrm{~K}$ soldering iron, with a single clear absorption line. The hole in the emission spectrum does not conform to any absorption lines in the atmosphere, so it is probably due to the rust/oxide layer on the surface of the soldering iron

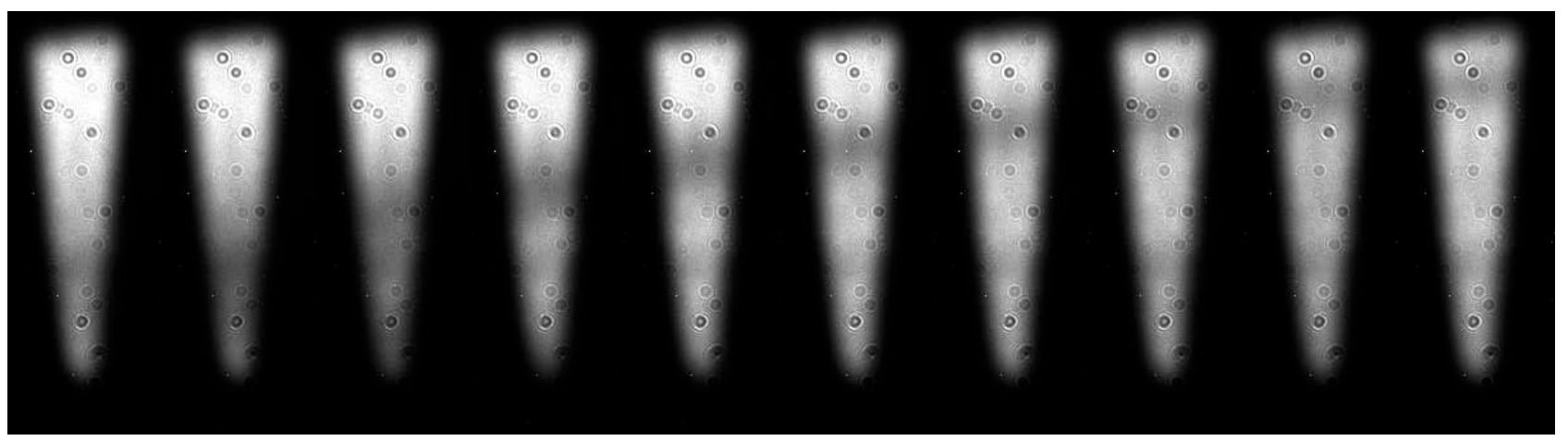

Figure 5. These images show the tip of a $700 \mathrm{~K}$ soldering iron, with the mixing wavelength scanning from 1049 to $1056.2 \mathrm{~nm}$. The images show the upconverted blackbody radiation in the range from 2.8 to $3.1 \mu \mathrm{m}$. As expected, the blackbody radiation is almost constant in this range, although we found a small hole in the emission spectrum, which moves across the tip as the mixing wavelength changes. This spectral hole probably comes from the oxide on the surface of the soldering iron (The stationary small particles in the images are dust particles on one of the lenses).

\section{FUTURE WORK}

The results presented in this paper are a work in progress. The tunable laser currently used as mixing source does not deliver enough power for rapid image acquisition, and it cannot tune fast enough, to allow for fast acquisition of multispectral images. Both of these concerns will be alleviated by changing the design of the setup.

The current design of the laser is not compatible with intracavity enhancement of the upconversion process, but we are exploring alternative designs for setting up a compact, fast and field deployable system.

\section{REFERENCES}

[1] Dam J. S., Tidemand-Lichtenberg P., Pedersen C., "Room-temperature mid-infrared single-photon spectral imaging," Nature Photonics 6, 788-793 (2012).

[2] Dam J. S., Pedersen C. and Tidemand-Lichtenberg P., "Improved space bandwidth product in image upconversion," Nonlinear Frequency Generation and Conversion: Materials, Devices, and Applications XI, 8240, p. 82400F, (2012).

[3] Midwinter J. E., "Image conversion from $1.6 \mu$ to the visible in lithium niobate," Appl. Phys. Lett. 12, 68-70 (1968).

[4] Hu Q., Dam J. S., Pedersen C., and Tidemand-Lichtenberg P., "High resolution mid-IR spectrometer based on frequency upconversion, "Opt. Lett. 37, 5232-5234 (2012).

[5] Jundt D., "Temperature-dependent Sellmeier equation for the index of refraction, ne, in congruent lithium niobate," Opt. Lett. 22, 1553-1555 (1997).

[6] Dam J. S., Tidemand-Lichtenberg P., Pedersen C., "Theory for upconversion of incoherent images," Opt. Express 20, 1475-1482 (2012). 